\title{
The Application of Grey System Model in AIDS Prevention and Control in Yunnan Province of China
}

\author{
Yang Baiyun \\ Yunnan Police College School of Information Network Security, Kunming, Yunnan, China
}

\section{Email address:}

bluebaiyun@sina.com

\section{To cite this article:}

Yang Baiyun. The Application of Grey System Model in AIDS Prevention and Control in Yunnan Province of China. Science Innovation. Vol. 4, No. 2, 2016, pp. 40-44. doi: 10.11648/j.si.20160402.13

Received: February 22, 2016; Accepted: March 16, 2016; Published: April 8, 2016

Abstract: Yunnan province is one of the most serious regions of HIV/AIDS epidemic in China. By October 31, 2015, total 29652 AIDS patients, 23316 deaths. Using the gray system model to predict the HIV/AIDS epidemic situation is a beneficial attempt. We can apply mathematical model in the study of this epidemic, managing to find its regularity and to predict its trend. Through the analysis of HIV/AIDS epidemic, we can lay a solid foundation for further research and work on AIDS prevention and control, and provide a reference basis for prevention strategies and countermeasures.

Keywords: Grey System Model, AIDS, Prevention and Control

\section{灰色系统模型在中国云南省艾滋病防控中的应用研究}

\section{杨白云}

云南警官学院信息网络安全学院, 昆明, 云南, 中国

\section{邮箱}

bluebaiyun@sina.com

摘要: 云南省是中国AIDS流行最严重的地区, 以2015年10月31日为时间结点, 统计资料显示, 云南省累计AIDS病人 29652 例, 死亡人数 23316 例。用灰色系统模型来预测HIV/AIDS流行态势是一种有益的尝试。通过对HIV/AIDS疫情的分析, 为 深入开展艾滋病预警奠定基础，为制订艾滋病防治策略与措施提供参考依据。

关键词: 灰色系统模型, 艾滋病, 防控

\section{1. 引言}

艾滋病是当前全球最关注的公共卫生问题之一。最新 资料显示，到2015年10月31日，中国云南省累计AIDS病人 29652例, 死亡人数23316例。中国云南省防控AIDS形势十 分严峻。

在有关艾滋病防控的研究中, 人们普遍采用定性分析 方式。如中国云南警官学院沙莉教授的中国国家社科课题 《高危群体艾滋病预防干预的宽容策略研究》, 批准立项
号: 07XSH005; 中国云南警官学院王清教授的中国国家社 科课题《流动人口艾滋病社区干预研究》, 批准立项号: 08CSHD21。本文是在参加上述两个社科课题工作的基础上, 申报完成的2015年中国云南省教育厅科学研究基金项目 《灰色系统模型在防控云南省艾滋病中的应用研究》的研 究成果。(验字 [2015]464号)

经资料查询, 除中国地区以外, 在艾滋病防控研究中 引入灰色系统理论进行研究还未见报道。查阅中国近年来 研究相关文献, 参考文献 [1] [2] [3] [4] 从不同侧面予以研 
究。在这些研究中存在的不足, 主要是只进行定性研究, 因为没有定量描述, 难免可能不够深刻。用灰色系统模型 来研究的文章还不多见, 参考文献 $[5]$ 给出。

\section{2. $\mathrm{GM}(1,1)$ 模型}

少数据、小样本、信息不完全和经验缺乏的不确定性 的系统称为灰色系统 [6]。灰建模是少数据的建模, 其目 的是在数据有限 (即有限序列) 的条件下, 模仿微分方程 建立具有部分微分方程性质的模型。灰预测是建立 (行为) 时轴上现在与未来的定量关系。通过此定量关系 (灰模型) 预测事物的发展。GM $(1,1)$ 是一个单变量的一阶微分方程 模型 [7], 利用它我们可以进行连续的动态预测。

设有 原始非负序列 $\mathrm{X}^{(0)}, \mathrm{X}^{(0)}=\left\{\mathrm{X}^{(0)}(1), \mathrm{X}^{(0)}(2), \cdots \mathrm{X}^{(0)}(\mathrm{n})\right\}$

$\mathrm{X}^{(1)}$ 为 $\mathrm{X}^{(0)}$ 的一阶累加生成序列,

$X^{(1)}=\left\{x^{(1)}(1), x^{(1)}(2), \cdots x^{(1)}(n)\right\}$

其中, $x^{(1)}(k)=\sum_{i=1}^{k} x^{(0)}(i), k=1,2, \ldots n$

建立白化形式的微分方程如式 (1)

$$
\frac{d x^{(1)}}{d t}+a x^{(1)}=b
$$

若 $\hat{\mathrm{a}}=[\mathrm{a}, \mathrm{b}]^{\mathrm{T}}$ 为参数列, 则有 $\mathrm{Ba}=\mathrm{Y}$

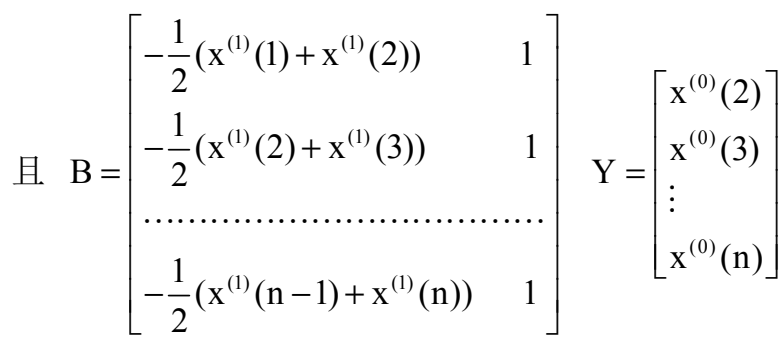

按最小二乘原理, 解得

$$
\hat{a}=\left(B^{T} B\right)^{-1} B^{T} Y
$$

求出参数解微分方程式 (1), 得到预测模型, 即GM $(1,1)$ 模型的时间响应序列为:

$$
\hat{x^{(1)}}(k+1)=\left(x^{(0)}(1)-\frac{b}{a}\right) e^{-a k}+\frac{b}{a} ; k=1,2, \ldots n
$$

还原值

$$
\begin{aligned}
\hat{x^{(0)}}(\mathrm{k}+1) & =\hat{x^{(1)}}(\mathrm{k}+1)-\mathrm{x}^{(1)}(\mathrm{k}) \\
& =\left(1-\mathrm{e}^{\mathrm{a}}\right)\left(\mathrm{x}^{(0)}(1)-\frac{\mathrm{b}}{\mathrm{a}}\right) \mathrm{e}^{-\mathrm{ak}} ; \mathrm{k}=1,2, \ldots \mathrm{n}
\end{aligned}
$$

$\operatorname{GM}(1,1)$ 模型中的参数 $\mathrm{a}$ 为发展系数, $\mathrm{a}$ 反映了 $\mathrm{x}^{\hat{(1)}}$ 及 $\mathrm{x}^{(0)}$ 的发展态势。a较小时, 模型精度较高。 $b$ 为灰色作 用量。

模型的精度由平均相对误差 $\bar{\Delta}$ 来刻画。给定 $\alpha$, 当 $\bar{\Delta}<\alpha$ 且 $\Delta_{n}<\alpha$ 成立时, 称模型为残差合格模型。其中残 差为:

$$
\varepsilon(\mathrm{k})=\mathrm{x}^{(0)}(\mathrm{k})-\mathrm{x}^{\hat{(0)}}(\mathrm{k})
$$

$\left(\mathrm{x}^{(0)}(\mathrm{k})-\right.$--实际数据; $\mathrm{x}^{(0)}(\mathrm{k})^{---}$模拟数据 $)$ 相对误差 (\%) 为:

$$
\Delta_{\mathrm{k}}=\frac{|\varepsilon(\mathrm{k})|}{\mathrm{x}^{(0)}(\mathrm{k})}
$$

平均相对误差 $\bar{\Delta}$ 为: $\bar{\Delta}=\frac{1}{\mathrm{n}} \sum_{\mathrm{k}=1}^{\mathrm{n}} \Delta_{\mathrm{k}}$

表1 精度检验等级参照表。

\begin{tabular}{ll}
\hline 精度等级 & 指标临界值 \\
\cline { 1 - 2 } 一极 & 相对误差 $\alpha$ \\
二级 & 0.01 \\
三级 & 0.05 \\
四级 & 0.10 \\
\hline
\end{tabular}

\section{3. 模拟演算}

说明: 以下模拟演算所使用数据资料来源均为中国云 南省疾病控制中心所发布。

\section{1. AIDS病死亡人数模型（5数据）}

表2 中国云南省历年累积AIDS死亡人数 [8]。

\begin{tabular}{llllll}
\hline 年份 & 2010 年 & 2011年 & 2012年 & 2013年 & 2014年 \\
\hline AIDS死亡 & 11609 人 & 14340 人 & 17268人 & 20093人 & 23316人 \\
\hline
\end{tabular}

用最近五年的数据, 代入式 (2) 计算 可得

$\mathrm{a}=-0.158361, \mathrm{~b}=11564.934741, \frac{\mathrm{b}}{\mathrm{a}}=-73029.102948$ [9]

表3 误差检验表。

\begin{tabular}{lllll}
\hline 序号 & 实际数据 $\mathrm{x}^{(0)}(\mathrm{k})$ & 模拟数据 $(4) \mathrm{x}^{\hat{(0)}}(\mathrm{k})$ & 残差 $(5) \varepsilon(\mathrm{k})=\mathrm{x}^{(0)}(\mathrm{k})-\mathrm{x}^{(0)}(\mathrm{k})$ & 相对误差 $(\%)(6) \Delta_{\mathrm{k}}=\frac{|\varepsilon(\mathrm{k})|}{\mathrm{x}^{(0)}(\mathrm{k})}$ \\
\hline $2(2011$ 年 $)$ & 14340 & 14522.93 & -182.94 & $1.276 \%$ \\
3 (2012年) & 17268 & 17014.90 & 253.09 & $1.466 \%$ \\
$4(2013$ 年 $)$ & 20093 & 19934.47 & 158.53 & $0.789 \%$ \\
5 (2014年) & 23316 & 23354.99 & 38.999 & $0.167 \%$ \\
\hline
\end{tabular}


平均 相 对 误 差 : $\bar{\Delta}=\frac{1}{4} \sum_{k=2}^{5} \Delta_{k}=0.924 \%<0.10$, 且 $\Delta_{5}=0.167 \%$ ；模型精度 为一级。

时间响应函数如式 (3) 所示为:

$\mathrm{x}^{\hat{(1)}}(\mathrm{k}+1)=84638.10948 \exp (0.158361 * \mathrm{k})-73029.10$

还原值如式 (4), 见表 3 (模拟数据 $\left.\mathrm{x}^{(0)}(\mathrm{k})\right)$ 。

从模型给出结的果来看, 平均相对误差仅为 $0.92 \%$, 说明模拟的精度很高。特别是 2014 年的数据中, 实际值与 模拟值误差仅为 38 人, 精度达到 $0.167 \%$, 可以说相当准确 了。死亡人数的模拟精度很高, 究其原因是艾滋病的治疗 目前也没有什么特别有效的方法, 治疗的效果近几年来也 没有什么根本的改变, 治疗效果对模型数据的影响较小, 所以用GM $(1,1)$ 模型得出的结果精度很高。用此模型来预 测随后3年的情况, 得到: 2015年中国云南省AIDS病死亡 人数累计预测值为 27362 人; 2016 年AIDS病死亡人数累计
预测值为 32057 人; 2017 年的AIDS病死亡人数累计预测值 为 37558 人。

从中国云南省艾滋病局通报的最新数据来看, 到 2015 年 10 月 31 日止, 中国云南省死亡艾滋病人数为 26510 人, 而我们的预测值为 27362 人, 模拟值与实际通报人数仅仅 相差 852 人, 误差值为 $3.2 \%$, 再一次说明我们的模拟是非 常有效的。

\section{2. HIV感染者模型（5数据）}

表4 中国云南省HIV累积统计数据。

\begin{tabular}{llllll}
\hline 年份 & 2010 年 & 2011年 & 2012年 & 2013年 & 2014年 \\
\hline $\begin{array}{l}\text { HIV 感 } \\
\text { 染者 }\end{array}$ & 62233 人 & 67869 人 & 73397人 & 56763人 & 50263人 \\
\hline
\end{tabular}

用最近五年的数据, 代入式 (2) 计算 可得

$$
\mathrm{a}=0.107272, \mathrm{~b}=82997.532121, \frac{\mathrm{b}}{\mathrm{a}}=773710.973363
$$

表5 误差检验表。

\begin{tabular}{lllll}
\hline 序号 & 实际数据 $\mathrm{x}^{(0)}(\mathrm{k})$ & 模拟数据 $(4) \hat{x}^{(0)}(\mathrm{k})$ & 残差 $(5) \varepsilon(\mathrm{k})=\mathrm{x}^{(0)}(\mathrm{k})-\mathrm{x}^{(0)}(\mathrm{k})$ & 相对误差 $(\%)(6) \Delta_{\mathrm{k}}=\frac{|\varepsilon(\mathrm{k})|}{\mathrm{x}^{(0)}(\mathrm{k})}$ \\
\hline 2 (2011年) & 67869 & 72370.61 & -4501.62 & $6.63 \%$ \\
3 (2012年) & 73397 & 65009.17 & 8387.83 & $11.43 \%$ \\
4 (2013年) & 56763 & 58396.52 & -1633.52 & $2.88 \%$ \\
5 (2014年) & 50263 & 52456.5 & -2193.51 & $4.36 \%$ \\
\hline
\end{tabular}

平均 相 对 误 差 : $\bar{\Delta}=\frac{1}{4} \sum_{k=2}^{5} \Delta_{k}=6.325 \%<0.10$, 且 $\Delta_{5}=4.36 \%$; 模型精度为 三级。

时间响应函数如式 (3) 所示:

$$
\mathrm{x}^{\hat{(1)}}(\mathrm{k}+1)=-711477.97 \exp (-0.107272 * \mathrm{k})+773710.97
$$

还原值如式 (4), 见表 5 (模拟数据 $\left.\mathrm{x}^{(0)}(\mathrm{k})\right)$ 。

从模型得出的结果来看, 平均相对误差是 $6.325 \%$, 模 型模拟的精度较高, 可以接受。而其中的2013年、2014 年实际数据比模拟数据低, 原因是由于中国云南省采取综 合防控措施, 大大降低了HIV感染者人数。用此模型来预
测随后 3 年的情况, 可得 2015 年为 47120 人, 2016 年为 42327 人， 2017 年为 38022 人。

\section{3. AIDS病病人模型（5数据）}

\begin{tabular}{|c|c|c|c|c|c|}
\hline 年份 & 2010年 & 2011年 & 2012年 & 2013年 & 2014年 \\
\hline $\begin{array}{l}\text { AIDS 病 } \\
\text { 人 }\end{array}$ & 20072人 & 25698人 & 31584人 & 26285人 & 29652人 \\
\hline
\end{tabular}

表6 中国云南省历年累积AIDS病人人数。

用最近五年的数据, 代入式 (2) 计算 可得

$$
a=-0.022779, b=26576.741006, \frac{b}{a}=-1166742.463965
$$

表7 误差检验表。

\begin{tabular}{llll|l}
\hline 序号 & 实际数据 $\mathrm{x}^{(0)}(\mathrm{k})$ & 模拟数据 $(4) \hat{x^{(0)}(\mathrm{k})}$ & 残差 $(5) \varepsilon(\mathrm{k})=\mathrm{x}^{(0)}(\mathrm{k})-\mathrm{x}^{(0)}(\mathrm{k})$ & 相对误差 $(\%)(6) \Delta_{\mathrm{k}}=\frac{|\varepsilon(\mathrm{k})|}{\mathrm{x}^{(0)}(\mathrm{k})}$ \\
\hline 2 (2011年) & 25698 & 27334.20 & -1646.20 & $6.41 \%$ \\
3 (2012年) & 31584 & 27974.21 & 3609.78 & $11.43 \%$ \\
4 (2013年) & 26285 & 28618.73 & -2333.74 & $8.88 \%$ \\
5 (2014年) & 29652 & 29278.11 & 373.89 & $1.26 \%$ \\
\hline
\end{tabular}


平均 相 对 误差为: $\bar{\Delta}=\frac{1}{4} \sum_{k=2}^{5} \Delta_{k}=6.994 \%<0.10$, 且 $\Delta_{5}=1.26 \%$; 模型精度为 三级。

时间响应函数如式 (3) 所示:

$\hat{\mathrm{x}^{(1)}}(\mathrm{k}+1)=1186814.46 \exp (0.22779 * \mathrm{k})-1166742.46$

还原值如式 (4), 见表 7 (模拟数据 $\left.\mathrm{x}^{(0)}(\mathrm{k})\right)$ 。

从模型模拟效果来看，平均相对误差是 $6.99 \%$, 模型 精度为三级。推测2015年、2016年、2017年的情况，其结 果: 2015 年中国云南省AIDS病人人数累计预测值为 29952 人; 2016 年累计预测值为 30642 人; 2017 年累计预测值为 31348人。

从中国云南省艾滋病局通报的最新数据来看, 到 2015 年 10 月 31 日止, 中国云南省艾滋病人实际通报人数为
33412 人, 与我们的预测值 29952 人相比较, 模拟值与实际 通报人数相差 3460 人, 误差率为 $10.4 \%$, 足以说明我们的 模拟还是比较有效的。

\section{4. AIDS病人及HIV感染者模型（5数据）}

表8 中国云南省历年累积AIDS病人及感染艾滋病病毒 (HIV) 人数。

\begin{tabular}{llllll}
\hline 年份 & 2010年 & 2011年 & 2012年 & 2013年 & 2014年 \\
\hline $\begin{array}{l}\text { 病人及 } \\
\text { 感染者 }\end{array}$ & 82305 人 & 93567人 & 104981人 & 83048 人 & 79915 人 \\
\hline
\end{tabular}

用最近五年的数据, 代入式 (2) 计算 可得

$$
\mathrm{a}=0.067267, \mathrm{~b}=108601.844908, \frac{\mathrm{b}}{\mathrm{a}}=1614488.316128
$$

表9 误差检验表。

\begin{tabular}{lllll}
\hline 序号 & 实际数据 $\mathrm{x}^{(0)}(\mathrm{k})$ & 模拟数据 $(4) \hat{\mathrm{x}^{(0)}(\mathrm{k})}$ & 残差 $(5) \varepsilon(\mathrm{k})=\mathrm{x}^{(0)}(\mathrm{k})-\mathrm{x}^{(0)}(\mathrm{k})$ & 相对误差 $(\%)(6) \Delta_{\mathrm{k}}=\frac{|\varepsilon(\mathrm{k})|}{\mathrm{x}^{(0)}(\mathrm{k})}$ \\
\hline 2（2011年） & 93567 & 99675.41 & -6108.41 & $6.528 \%$ \\
3 (2012年) & 104981 & 93191.08 & 11789.92 & $11.231 \%$ \\
4 (2013年) & 83048 & 87128.58 & -4080.58 & $4.914 \%$ \\
5 (2014年) & 79915 & 841460.47 & -1545.47 & $1.934 \%$ \\
\hline
\end{tabular}

平均相对误差为: $\bar{\Delta}=\frac{1}{4} \sum_{k=2}^{5} \Delta_{k}=6.152 \%<10 \%$, 且 $\Delta_{5}=1.934 \%$; 模型精度为三级。时间响应函数如式 (3) 所示:

$$
\mathrm{x}^{\hat{(1)}}(\mathrm{k}+1)=-1532183.316128 \exp (-0.067267 * \mathrm{k})+1614488.316
$$

还原值如式 (4), 见表 9 (模拟数据 $\left.\mathrm{x}^{(\hat{0})}(\mathrm{k})\right)$ 。

从模拟结果看, 平均相对误差 $6.15 \%$, 模型精度三级, 可信。2013年、2014年的实际数据低于模拟数据, 其原因 在于中国云南省采取的各种干预措施到位, 大大降低了感 染人数。用模型来预测 2015 年、2016年、2017年中国云南 省AIDS病人及HIV感染者人数情况, 得到: 2015 年预测值 为76171人, 2016 年累计预测值为 71206 人, 2017 年累计预 测值为 66574 人。

\section{2014年、2015年中国云南省艾滋病疫情特点 及防控工作}

\section{1 . 疫情特点}

2014年1-10月，中国云南省报告HIV和AIDS病人 9601 例, 其中HIV感染6857例、AIDS病人 2744 例, 死亡 2184 例。[10]

2015年1-10月, 中国云南省报告HIV感染9768例, AIDS 病人 2893 例, 死亡 2283 例。[11]

\section{1. 1. 性传播成主要渠道}

2014年10月性传播比例达到 $89.5 \%$ ，已经成为中国云 南省艾滋病传播的最主要途径。其中 $82.6 \%$ 为婚外不安全 性行为传播, 这当中约 $80 \%$ 为商业性行为传播; $4.7 \%$ 为男 男性行为传播, 男男性行为传播呈上升趋势。12.7\%为家 庭内配偶间传播。

2015年性传播达到 $91.4 \%$, 同比增长了 $1.9 \%$ 。

\section{1. 2. 青年学生报告数增加}

2014年1-10月报告的73例青年学生HIV感染者中, 男 性61例, 女性12例。

2015年报告的青年学生HIV感染者94例, 比2014年增 长 $28.8 \%$ 。其中同性传播的比例高达 $54.8 \%$, 逐年上升趋势 显著。

\section{1. 3. 老年人报告数增加}

2014年1-10月，新报告的HIV感染者和AIDS病人中， 60 岁以上老年人占比 $12.0 \%$; 2015 年 60 岁以上老年人占比 $13.7 \%$, 同比增长了 $1.7 \%$ 。 


\section{2. 防控工作}

中国云南省在防控艾滋病中做了大量卓有成效的工 作：一是广泛开展更有针对性和实效性宣传教育; 二是加 大监测检测，2014年1-10月中国云南省共开展HIV抗体检 测 610.9 万人份; 三是加强病源管理; 四是开展行为干预, 2014年1-3季度中国云南省共发放安全套4435万只, 2014 年1-10月中国云南省暗娼月均干预 21589 人, 男男性行为 者月均干预 5335 人; 五是开展母婴阻断, 母㚣传播阻断覆 盖率达 $100 \%$ 。 六是开展抗病毒治疗, AIDS抗病毒治疗患 者的病死率下降到了 $2.2 \%$, 低于中国平均水平; 七是加大 关怀救助力度, 2014年对纳入城市低保的13031名艾滋病 病毒感染者/病人和纳入农村低保的9464名HIV感染者/病 人共救助 4387.9 万元。 [12]

\section{5. 防控策略}

通过用灰色系统模型对中国云南省艾滋病疫情模拟, 我们看到, 中国云南省在艾滋病防控上的形势非常严峻。

\section{1. 防治难点}

一是艾滋病传播渠道从高危人群向一般人群扩散成 为新常态，防控难度大大增加，性传播比例超过 $90 \%$, 传 播方式更加隐蔽, 防控形势更加严峻; 二是由于人员流动 性大, 男同、暗娼和吸毒人群在各城市集中, 感染者基数 庞大，防治管理难度大大增加; 三是艾滋病综合防治示范 社区对于在流动人口、老年人、青少年等重点人群中开展 防艾宣传和干预措施单一, 动员检测、行为干预、感染者 管理和转介治疗工作不足。

\section{2. 对策}

一、继续坚持政府主导, 确保长效工作机制良好运行。 坚持执行防治艾滋病工作 “一把手” 负责制和责任目标考 核制, 把防治艾滋病工作作为各级党委、政府的一项政治 任务。

二、健全并完善工作保障机制。经费保障: 实验室能 力建设、行为干预、感染者管理、治疗、母婴阻断、培训; 人员数量不足: 争取编制 + 到位到岗 + 临时聘用人员 + 社区 组织+志愿者; 队伍不稳定: 落实艾滋病防治工作人员和 职业暴露高危人员岗位补贴，建立激励机制。

三、进一步创新社会管理, 加大政府购买社会组织防 治艾滋病服务力度。

四、继续加强艾滋病综合防治示范区建设工作，继续 扩大试点社区的建设, 充分发挥基层社区的积极作用。

五、继续推进扩大检测、扩大治疗工作。全面推行抗 病毒治疗 “一站式服务” 经验。

六、公安、计生、民政、教育、社区街道办、司法、 卫生多部门合作，控制艾滋病经性途径传播蔓延。

七、加强边境地区防艾工作。制定了《中国云南省边 境地区艾滋病防治项目工作方案（2014一-2018年）》。

\section{6. 结论}

应用灰色模型模拟得出的数据, 近期值比较准确, 有 较大参考价值。中、远期预测可以反映出一种发展趋势。 从模型模拟数据来看, 中国云南省在防控艾滋病的道路上 还有漫漫征程, 形势不容乐观。刚刚过去的2015年是中国 云南省第三轮禁毒防艾人民战争的收官之年, 从中国云南 省艾滋病局通报的最新数据来看, 到2015年10月31日止, 中国云南省存活艾滋病病毒感染者 87634 人, 艾滋病病人 33412 人, 死亡艾滋病人数为 26510 人 [13], 触目惊心的数 据再一次警醒我们, 中国云南省的防控工作任务繁重而艰 巨。

\section{参考文献}

[1] 陆林. 云南艾滋病流行 20 年. [J]. 昆明医科大学学报 2013, (6) :1-4

[2] 陈东等. 我国艾滋病疫情发展趋势预测的防控措施分析 [J]. 中国公共卫生管理 2013, (1) : 3-7

［3］肖民扬等. 云南省艾滋病病毒感染者和艾滋病患者的高危 行为影响因素分析 $[J]$. 中华疾病控制杂志 2013 , (12) : 1021-1024

[4] 安晓静等. 云南省艾滋病综合管理对相关服务可及性的影 响分析 $[J]$. 中国艾滋病性病2013, (2) : 134-137

[5] GM $(1,1)$ 模型在预测云南省艾滋病病毒 (HIV) 感染者上的应 用研究. [J]. 云南大学学报 (自然科学版) 2008 , 30 (S1) : $452-455$

[6] 邓聚龙. 灰色系统基本方法 [M]. 武汉: 华中科技大学出版社, 2004: 2

[7] 刘思峰, 党耀国, 方志耕. 《灰色系统理论及其应用》 [M] (第三版) 科学出版社, 2007年1月:164

[8] 云南疾控资讯网, http://www. yncdc. cn

[9] 刘斌. 灰色建模系统 (软件), 科学出版社, 2004年

[10２014年 10 月 31 日：云南省艾滋病疫情， http://www. yncdc. cn/newsview. aspx?id=107070

[11] 今年1-10月云南新增染艾患者9768例. 春城晚报A042015、 12、 1

[12] 2014 年云南省艾滋病主要防治工作进展, http://www. yncdc. cn/newsvi ew. aspx?id=107071

[13] 今日数据. 春城晚报A042015、12、1 